\title{
Drop Call Probability in Established Cellular Networks: from data Analysis to Modelling
}

\author{
G. Boggia, P. Camarda, A. D’Alconzo, A. De Biasi and M. Siviero \\ DEE - Politecnico di Bari, Via E. Orabona, 4 - 70125 Bari (Italy) \\ e-mail: \{g.boggia, camarda, a.dalconzo, a.debiasi\}@poliba.it, massimo.siviero@vodafone.com
}

\begin{abstract}
The ever increase of advanced services offered by modern cellular networks require stringent Quality of Service $($ QoS) guarantee, obtained as the typical result of many optimization procedures. In this paper the phenomenon of dropped calls, one of the most important indices of QoS in a large scale well-established cellular network, has been analyzed. We verified from measured data traffic that, in a well-established cellular network, models available in literature are useless to pursue the objective of service optimization: many phenomena, neglected till now, heavily influence the call termination. To relate the drop call probability to these phenomena, an original analytical model has been developed. The obtained results, validated by experimental measures taken from a real network, can allow the network operator to optimize system performance improving the offered Quality of Service and their revenue.
\end{abstract}

\section{INTRODUCTION}

The drop call probability is one of the most important Quality of Service (QoS) index used to monitor the performance of cellular networks. Its minimization is the typical result of many optimization procedures applied to several service aspects as: the maximization of service coverage area and of network usage, the minimization of interference and congestion, the optimum traffic balancing among the different frequency layers (e.g., 900 and $1800 \mathrm{MHz}$ in the European GSM standard).

For these reasons, the drop call probability has been the subject of several studies [1] - [8]. Generally, in the models developed in these papers, appropriate cell and system planning, taking into account propagation conditions in the service area, are considered. Moreover, the fundamental hypothesis that calls are terminated only due to handover failure is assumed (see for example, [2], [7]); that is, an active user can change cell several times and, during one of these transitions between cells, his connection can be dropped. Call termination caused by handover failure depends, apart from system characteristics, on user behaviour; therefore, it is an event non directly predictable by system planners.

The evolution of these models concerns the release of some restrictive hypotheses, such as a more general modelling of Call Holding Time or Cell Residence Time, initially assumed exponentially distributed [4], [6], [9], or the classification of users in different classes to take into account different mobility and services [10], or a different modelling of the cells assumed as service centers with a finite user population [11].

Although there is a vast literature on this subject, the analysis of measured data traffic, supplied by Vodafone Italy and covering a part of the southern Italian territory, allowed us to verify that, in a well-established cellular network, previous models are useless to pursue the objective of service optimization. In fact, observing the behavior of actual wireless cellular networks, the handover failure is usually a negligible event. So that, many other phenomena become much more relevant in influencing the call dropping; in particular, call termination is mainly due to propagation conditions, irregular user behavior, and so on.

Modelling these phenomena is quite interesting both from theoretic and practical point of views. In fact, the knowledge of call dropping behaviour as a function of some network parameters (e.g., traffic load, call duration, and so on) can help operators to optimize performance and then to improve QoS and their revenue.

Therefore, in this paper a simple new drop call probability model, which is integrated in an experimental context, has been developed, with specific attention to the model validation on a real GSM network. The data traffic have been exploited in two ways. First we studied the call duration statistic distributions, the phenomena that lead to call termination, the characterization of both call arrival process and call drop process. As a second aspect, these data drive us in formulating the analytical model and were useful for a comparison with the results obtained from the proposed model. Even if we validated the model with GSM data, the approach is more general. Following a similar procedure, model parameters can be easily derived from measured data in other cellular systems (e.g., UMTS cellular networks) and, then, applied to performance prediction.

The rest of the paper is organized as follows. In section II the supplied measured data have been statistically analyzed. In section III the original analytical model has been developed evaluating the drop call probability, as a function of some network parameters. Numerical results are reported in section IV. Finally, some conclusions are drawn in section V.

\section{Data Collection And Analysis}

In this paper, several data have been collected for ten months from the Vodafone Italy GSM network: from February 2003 to December 2003. These data sets were related to ten GSM traffic cells, for a total of about 200,000 monitored calls, chosen as a representative sample to obtain numerically significant data. The sample significance is defined in terms of cell extension, number of served subscribers in the area, 
TABLE I.

Estimated Statistical PARAMETERS

\begin{tabular}{ll|ccc}
\hline & & $\hat{\mu}[s]$ & $\hat{\sigma}[s]$ & $C$ \\
\hline \hline \multirow{3}{*}{ Cell 1 } & Normal Call Duration & 121.74 & 205.65 & 1.69 \\
& Dropped Call Duration & 96.01 & 172.09 & 1.79 \\
& Inter-departure time & 92.44 & 87.67 & 0.95 \\
& Interarrival time & 6.14 & 6.14 & 1.00 \\
\hline \multirow{3}{*}{ Cell 2 } & Normal Call Duration & 93.20 & 152.18 & 1.63 \\
& Dropped Call Duration & 130.20 & 339.70 & 2.61 \\
& Inter-departure time & 67.72 & 78.23 & 1.16 \\
& Interarrival time & 6.60 & 6.54 & 0.99 \\
\hline \multirow{3}{*}{ Cell 3 } & Normal Call Duration & 100.97 & 134.89 & 1.34 \\
& Dropped Call Duration & 92.86 & 159.35 & 1.72 \\
& Inter-departure time & 101.08 & 103.33 & 1.02 \\
& Interarrival time & 3.18 & 3.53 & 1.11 \\
\hline \multirow{3}{*}{ Cell 4 } & Normal Call Duration & 111.15 & 187.50 & 1.69 \\
& Dropped Call Duration & 95.64 & 213.47 & 2.23 \\
& Inter-departure time & 85.01 & 94.28 & 1.11 \\
& Interarrival time & 6.54 & 7.01 & 1.07 \\
\hline \multirow{3}{*}{ Cell 5 } & Normal Call Duration & 108.35 & 198.13 & 1.83 \\
& Dropped Call Duration & 97.27 & 174.25 & 1.79 \\
& Inter-departure time & 99.65 & 101.27 & 1.01 \\
& Interarrival time & 4.00 & 5.00 & 1.25 \\
\hline
\end{tabular}

and traffic load; for this reasons, macro GSM cells in a urban metropolitan environment where chosen.

By using the Clear Codes reported in the databases of the network operator, calls are classified in Dropped and Not Dropped, distinguishing for the former the causes of dropping. By observing the Dropped and Not Dropped Calls, and the difference between their starting instants, it is possible to evaluate the distribution of the arrival process, which we refer to as interarrival process. Likewise, from the analysis of the difference between the ending times, it is possible to evaluate the distribution of inter-departure times for Dropped Calls. Moreover, we have analyzed the durations of normally terminated calls and dropped calls calculated as difference between the charging ending time.

First of all, a statistical analysis of the measured data was carried out, allowing us the estimation of mean $\mu$ and variance $\sigma^{2}$ of each process, by using the well known convergent and not polarized estimators for these parameters [12]. Moreover, we evaluate the coefficient of variation, $C$, defined as the ratio between standard deviation and mean; this parameter is an index of data dispersion around the mean value. In Table I estimated statistical parameters referred to 4 hours around the Busy Hour (i.e., the hour in which there is the maximum traffic load in the network) are reported for some cells. Given that the coefficient of variation takes into account the first and second order sample statistic, it allows us to make a first choice between the candidate statistical distribution to fit measured data. In particular, we observed that the durations of normally terminated calls and dropped calls show a value of $C$ greater than 1 , whereas the interarrival and the inter-departure times have a coefficient of variation $C \simeq 1$.

From this and, by taking also into account other known results about the lognormal distribution of the channel holding time (i.e., the time during which a channel in a cell is occupied by a call) [13], we made the hypothesis that call durations have a lognormal distribution whereas interarrival and interdeparture times have an exponential distribution. The analytical expressions of exponential and lognormal distribution are reported below [12]:

$$
\begin{aligned}
f_{X}(x) & =\lambda e^{-\lambda x}, & \lambda>0, x \geq 0, \\
g_{T}(t) & =\frac{1}{t \varphi \sqrt{2 \pi}} e^{-\frac{\ln t-\vartheta}{\varphi^{2}}}, & \varphi, \theta>0, t \geq 0 .
\end{aligned}
$$

It is worthwhile to note that this result is different from the one reported in [13], where a lognormal is used to fit the channel holding time for a single cell, while the call duration, considered in this paper, is the sum of all the channel holding times in the cells visited by the user during the same call.

To check these hypotheses, the chi-square goodness-of-fit test (or $\chi^{2}$-test) was used [12], [13], following the algorithm introduced by Fisher [14]. For the estimation of distribution parameters, the corresponding maximum likelihood estimators were used for both the exponential and lognormal distribution, which are respectively (see [12], [13]):

$$
\begin{gathered}
\hat{\lambda}=n / \sum_{i=1}^{n} T_{i}, \\
\hat{\vartheta}=\frac{1}{n} \sum_{i=1}^{n} \ln \left(t_{i}\right), \quad \hat{\varphi}=\frac{1}{n} \sum_{i=1}^{n} \ln \left(t_{i}\right)^{2}-\hat{\vartheta}^{2},
\end{gathered}
$$

where $T_{i}$ and $t_{i}$ are the time samples.

The tests were done using a significance level $\alpha=0.01$. They gave positive results in all the trials, both with cell data referred to 24 hours and with data for the same cell referred to 4 hours around the Busy Hour. As in [13], also in this work it was necessary to filter data samples which showed an anomalous relative frequency, but, whereas in [13] the $26 \%$ of the sample data were reject, in our analysis we never discard more than $5 \%$ of data.

The obtained results show that both durations of normally terminated calls and dropped calls are lognormal distributed. Moreover, our statistical analysis confirms the exponential hypothesis both for call arrival process and for the interdeparture process between consecutive dropped calls. Even if these results confirms some previous similar results [13], we highlight, however, that the analysis of dropped calls for both interdeparture time and call duration time does not appear in previous scientific papers.

As an example, in Fig. 1 the measured samples and the fitted lognormal distribution for the duration of normal terminated calls are reported, while in Fig. 2 the interarrival times between calls, are fitted by an exponential distribution.

In the analysis of experimental measures, it was studied the aforesaid dropped calls causes by means of the Clear Codes and by the correspondence tables between these codes and the related physical phenomena. In Table II, results for one cell are illustrated. The analysis was repeated referring to different periods of time and to different cells to exclude 


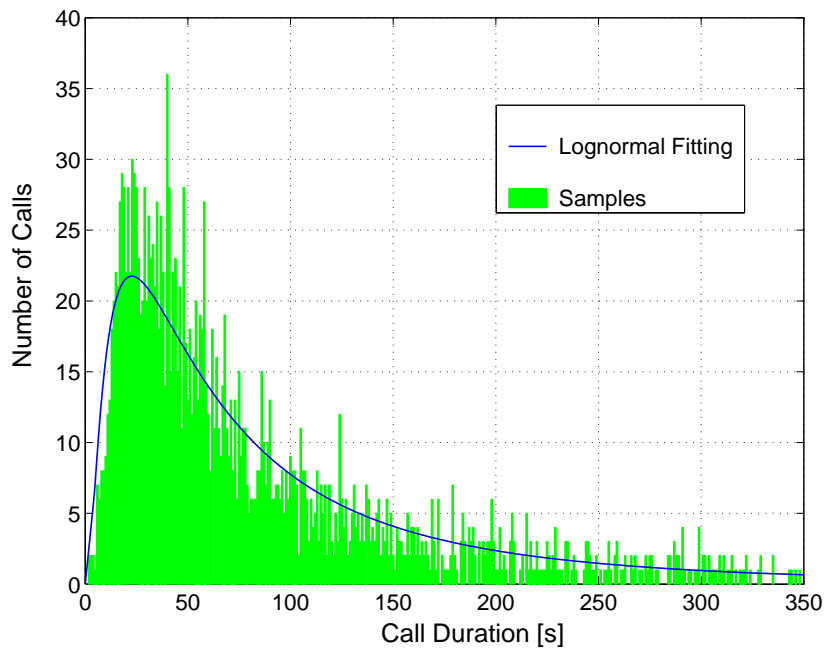

Fig. 1. Fitting of call duration with lognormal distribution (cell 1 observed for 4 hours).

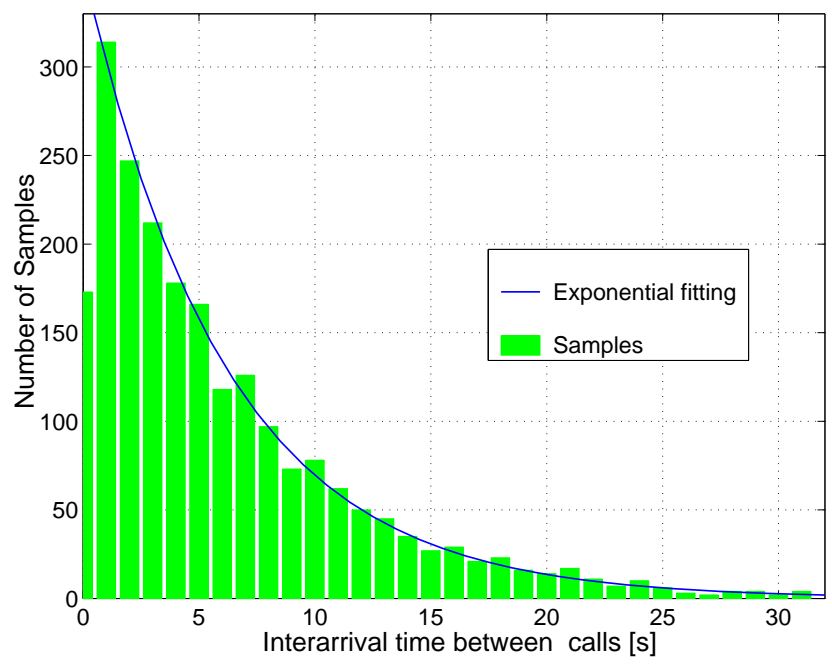

Fig. 2. Fitting of interarrival time between calls, with exponential distribution (cell 1 observed for 4 hours).

possible influence of local phenomena. The prevailing causes are the electromagnetic ones (e.g., power attenuation, deep fading). A lot of calls are dropped due to irregular user behaviour (e.g., mobile equipment failure, phone switched off after ringing, subscriber charging capacity exceeded during call). Other causes are due to abnormal network response (e.g., radio and signalling protocols error).

The main conclusion of drop cause analysis was that, in a well-established cellular network, was not possible to find a prevailing cause of call forced termination, rather a heterogeneous independent mix of causes. In particular, the handover failure is almost an unknown event in such environment thanks to the reliability and the effectiveness of the deployed handover control procedure. Our opinion is that the obtained results are not limited to the analyzed cells, but generally valid on the whole network.

The effort made in this paper was to model all the causes
TABLE II.

OcCurRence of CAll Dropping Causes

\begin{tabular}{lc}
\hline DROP CAUSES & OCCURRENCE [\%] \\
\hline \hline Electromagnetic causes & 51.4 \\
Irregular user behaviour & 36.9 \\
Abnormal network response & 7.6 \\
Others & 4.1 \\
\hline
\end{tabular}

of forced termination as a single phenomenon following the statistic of Poisson. In fact, thanks to the aforesaid exponential distribution of both the interarrival and departure times, and with the additional hypothesis of their independence, the Poissonian hypothesis for either the call arrival and call dropping process can be accepted.

\section{ANALYTICAL MODEL}

Starting from data analysis, we have developed an analytical model to predict the drop call probability as a function of network parameters.

Let $\lambda_{t}$ be the total traffic entering in the generic cell. If the call blocking probability due to absence of available traffic channels, $P_{b}$, is almost negligible (that is, the system can be considered as non blocking), then $\lambda_{t}$ is also the total traffic accepted in the cell. A fraction of this traffic will be dropped with probability $P_{d}$, that is the drop call probability, by phenomena described in the previous section; therefore the intensity $\nu_{d}$ of the Poisson process which models the drop call behaviour is given by:

$$
\nu_{d}=\lambda_{t} \cdot P_{d} .
$$

Now, we can consider the generic call behaviour (see Fig. 3): a call request is served by a generic channel, randomly selected, and the call will finish, if correctly terminated, after a duration time, $T$, which is a random variable with a lognormal p.d.f, $g_{c}(t)$, as we shown in the previous section. Otherwise, if one of the phenomena, which cause the call dropping, happens, the call will be dropped after a duration time, $T_{d}$, which is also a random variable, with a lognormal p.d.f., $g_{d}(t)$. Given that a Poisson process models the calls arrival, the cell can be modelled as a $\mathrm{M} / \mathrm{G} / \infty$ queue.

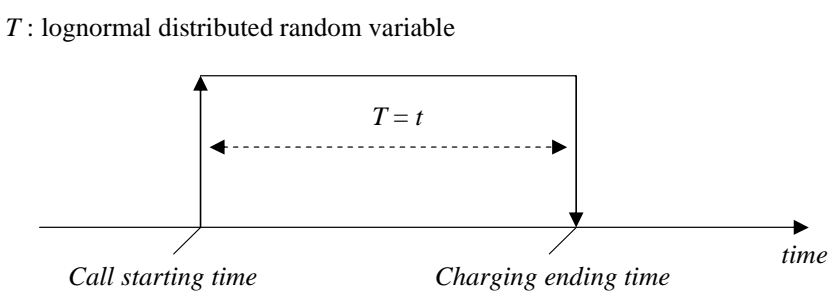

Fig. 3. Time diagram to describe cell behaviour.

The probability that a call, among $k$ active ones, is not involved by one single drop event, during the duration time $T=t$, is $(k-1) / k$; obviously, given that drop events are independent, this probability is $[(k-1) / k]^{n}$ if there are $n$ drop events. On the other hand, the inter-departure times, 
which are the drop call instants, constitute a Poisson process with parameter $\nu_{d}$. Let $Y$ be the random variable that counts the number of drops; thus, the probability that there are $n$ drops in $T=t$ is:

$$
P(Y=n)=\frac{\left(\nu_{d} t\right)^{n}}{n !} e^{-\nu_{d} t}, \quad n \geq 0 .
$$

Hence, by the total probability theorem, the probability $P(C \mid T=t, N=k)$ that a call is completed, with duration $T=t$ and when in the presence of $k$ contemporary calls, is:

$$
\begin{aligned}
P(C \mid T=t, N=k) & =\sum_{n=0}^{\infty}\left(\frac{k-1}{k}\right)^{n} \frac{\left(\nu_{d} t\right)^{n}}{n !} e^{-\nu_{d} t} d t \\
& =e^{-\nu_{d} t} \sum_{n=0}^{\infty} \frac{\left(\frac{k-1}{k} \nu_{d} t\right)^{n}}{n !} \\
& =e^{-\nu_{d} t} \cdot e^{\frac{k-1}{k} \nu_{d} t}=e^{-\frac{\nu_{d} t}{k}}
\end{aligned}
$$

Using again the total probability theorem, summing over all the possible numbers of contemporary active calls, the probability that a call is normally terminated with duration $t$ is:

$$
P(C \mid T=t)=\sum_{k=1}^{\infty} P(C \mid T=t, N=k) \cdot P(N=k)
$$

where $P(N=k)$ is the probability that in the cell there are $k$ active users.

As experimentally verified, in well-established cellular networks, operating in normal conditions, the dropping causes are not due to unavailability of communication channels. As a consequence, a generic call can be modelled as belonging to a $\mathrm{M} / \mathrm{G} / \infty$ queue. From queueing theory we have [15]:

$$
P(N=k)=c_{N} \cdot \frac{\rho^{k}}{k !}, \quad k \geq 1,
$$

where $\rho$ is the utilization factor, given by the product between the total traffic $\lambda_{t}$ and the mean service time $T_{c}$, and $c_{N}$ is a normalization coefficient which considers that there is at least one ongoing call during the time $T=t$.

Applying the normalization condition it follows that the coefficient $c$ is given by:

$$
c_{N}=\frac{1}{e^{\rho}-1},
$$

and substituting (10) in (9), we obtain:

$$
P(N=k)=\frac{1}{e^{\rho}-1} \cdot \frac{\rho^{k}}{k !}, \quad k \geq 1 .
$$

Now, substituting (11) and (7) in (8), we have:

$$
P(C \mid T=t)=\sum_{k=1}^{\infty} e^{-\frac{\nu_{d} t}{k}} \cdot \frac{1}{e^{\rho}-1} \cdot \frac{\rho^{k}}{k !} .
$$

\begin{tabular}{|c|c|c|c|}
\hline & $\begin{array}{c}\text { (by measures) } \\
P_{d}[\%]\end{array}$ & $\begin{array}{c}\text { (by model) } \\
P_{d}[\%]\end{array}$ & $\begin{array}{c}\text { Confidence Interval } \\
{[\%]}\end{array}$ \\
\hline Cell 1 & 6.79 & 6.52 & {$[5.84 ; 7.88]$} \\
\hline Cell 2 & 7.29 & 7.47 & {$[6.27 ; 8.46]$} \\
\hline Cell 3 & 3.07 & 3.12 & {$[2.61 ; 3.61]$} \\
\hline Cell 4 & 6.72 & 6.74 & {$[5.75 ; 7.84]$} \\
\hline Cell 5 & 4.04 & 4.00 & {$[3.44 ; 4.74]$} \\
\hline
\end{tabular}

Thus, it is straightforward to evaluate the probability of a normally terminated call, $P_{c}$, simply considering every possible call duration:

$$
P_{c}=\int_{0}^{\infty} P(C \mid T=t) g_{c}(t) d t=c_{N} \sum_{k=1}^{\infty} \frac{\rho^{k}}{k !} \int_{0}^{\infty} g_{c}(t) e^{-\frac{\nu_{d} t}{k}} d t .
$$

TABLE III

Drop CALl Probability Results

Finally, since the drop call probability is $P_{d}=\left(1-P_{c}\right)$, it results that:

$$
P_{d}=1-\frac{1}{e^{\rho}-1} \sum_{k=1}^{\infty} \frac{\rho^{k}}{k !} \int_{0}^{\infty} g_{c}(t) e^{-\frac{\nu_{d} t}{k}} d t .
$$

It is worth noticing that eq. (14) depends on the drop call rate $\nu_{d}$, the p.d.f. $g_{c}(t)$ of the duration of normally terminated calls, the average number $\rho$ of active users in the cell, and the total call arrival rate $\lambda_{t}$.

Finally, given that in developing our model there are no assumption on a particular technology, it is worthwhile to note that the model can be exploited to predict the drop call probability in different cellular networks (e.g., GSM, PCS, UMTS).

\section{RESUlTS}

In this section numerical results obtained with the developed model are reported. First, for each examined cell, we evaluated, from the measured data, an estimation of the call drop probability $P_{d}$, and its confidence interval with an usual confidence level $1-\alpha=0.95$ [12]. This is to establish the acceptance region for the analytical results, so that values coming from the analytical model are acceptable provided that they fall in that interval.

Then, the model has been validated by comparing drop call probability estimated by measured data with the ones evaluated with the developed analytical model. In Table III such a comparison is reported for some cells and it shows that in every case the analytical results fall in the call drop probability confidence interval. We found that this behaviour is verified for all sets of measured data. The results are more accurate for data sets referred to 4 hours around the Busy Hour, which nevertheless are the most interesting for network operator purpose.

A better agreement comparing drop call probabilities evaluated by model and by measured data could be obtained by using larger data sample [12]. In fact, as the data set get larger (for heavy traffic cells) both the confidence interval get smaller and the estimation of the analytical model input parameter $\left(\nu_{d}\right.$, $\lambda_{t}$, etc.) get more accurate, reducing errors coming out from the data statistical fluctuation.

The model can be exploited to evaluate network performance as a function of network parameters. We verified the correlation between $\nu_{d}$ and $\lambda_{t}$, i.e., if variation of $\lambda_{t}$ results in 
$\nu_{d}$ variation. From data we found out that a linear dependence law between these two parameters exists:

$$
\nu_{d}=m \lambda_{t}+b
$$

where $m$ and $b$ could be obtained with a least square regression tecnique [12]. Fig.4 shows that relatively great $\lambda_{t}$ variations produce only little $\nu_{d}$ changes.

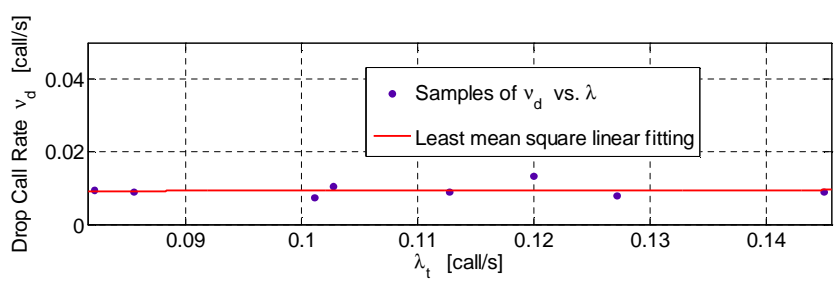

Fig. 4. Total traffic entering a cell, $\lambda_{t}$, vs. the drop call rate, $\nu_{d}$.

In Fig. 5 the drop call probability is reported as a function of the total traffic entering in the cell, $\lambda_{t}$, with several values of coefficient of variation. The mean call duration is assumed equal to $100 \mathrm{~s}$, near to the typical value observed in measured data (see Table I).

As shown in Fig.5, system performance improves as the traffic entering in the cell increases. Since $\nu_{d}$ varies according to eq. (15), increasing the traffic load, the number of dropped calls remains quite constant and, then, the drop call rate decreases.

The more interesting result coming out from Fig. 5 is the effect of coefficient of variation on drop call probability. This probability decreases as coefficient of variation increases; that is, fixing mean call duration, values more dispersed around this mean reduce call dropping probability. Similar results on other system performance are reported in literature [6] [16]. This behaviour can partially explain the performance improvement of some well-established mobile networks in which the presence at the same time of many services leads to a greater differentiation of call durations.

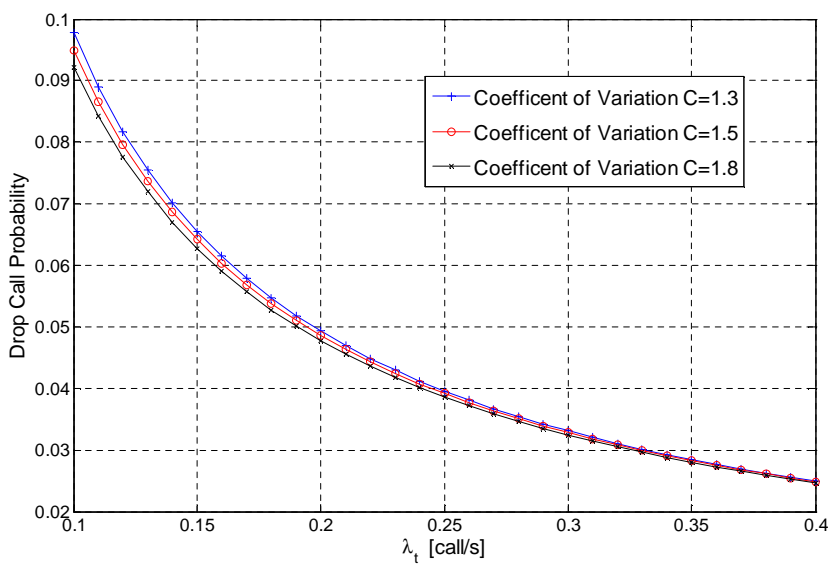

Fig. 5. Drop call probability vs traffic $\lambda_{t}$, with several coefficients of variation $C$.

\section{CONCLUSions}

In this paper, starting from the statistical analysis of data in a real cellular network, we have verified the lognormal hypothesis for distribution of the call holding time of both the normally terminated and the dropped calls. Moreover, the phenomena which cause the conversation interruption have been classified, verifying that handover failure become negligible in a well-established mobile telecommunication network. With both planning optimization and fine tuning of network parameters, several secondary phenomena (irregular users behaviours, abnormal network response, power attenuation, and so on) become non negligible and have been analytically modelled. The proposed new model has been validated comparing its results with data collected from a real cellular network, in a wide range of traffic load conditions.

The approach adopted in this paper can be fruitfully exploited to study different cellular networks with different distributions for the call duration, provided that eq. (14) can be numerically solved.

\section{REFERENCES}

[1] Y. Fang and I. Chlamtac, "A new model and its application in channel holding time characterization in PCS network," in Proc. INFOCOM'99, New York, March 1999.

[2] - "Teletraffic analysis and mobility modeling of PCS networks," IEEE Trans. Commun., vol. 47, no. 7, pp. 1062-1072, July 1999.

[3] D. Hong and S. Rappaport, "Traffic model and performance analysis for cellular mobile radio telephone systems with prioritized and nonprioritized handoff procedures," IEEE Trans. Veh. Technol., vol. VT-35, pp. 72-92, August 1986.

[4] Y. Fang, I. Chlamtac, and Y.-B. Lin, "Modeling PCS network under general call holding time and cell residence time distributions," IEEE/ACM Transaction Networking, vol. 5, no. 6, pp. 893-906, December 1997.

[5] — "Call performance for a PCS network," IEEE J. Select. Areas Commun., vol. 15, no. 8, pp. 1568-1581, October 1997.

[6] P. V. Orlik and S. Rappaport, "A model for teletraffic performance and channel holding time characterization in wireless cellular communication with general session and dwell time distributions," IEEE J. Select. Areas Commun., vol. 16, no. 5, pp. 788-803, June 1998.

[7] H. Zeng, Y. Fang, and I. Chlamtac, "Call blocking performance study for PCS networks under realistic mobility assumptions," Telecommunications Systems, vol. 19, no. 2, pp. 125-146, February 2002.

[8] M. Rajaratnam and F. Takawira, "Handoff traffic modeling in cellular networks," in Proc. IEEE GLOBECOM'97, Phoenix, AZ, November 1997.

[9] Y.-B. Lin and I. Chlamtac, "Effect of erlang call holding time on PCS call completion,” IEEE Trans. Veh. Technol., vol. 48, no. 3, pp. 815-823, May 1999.

[10] M. Rajaratnam and F. Takawira, "Nonclassical traffic and performance analysis of cellular mobile networks with and without channel reservation," IEEE Trans. Veh. Technol., vol. 49, no. 3, pp. 817 -834, May 2000.

[11] G. Boggia, P. Camarda, and N. D. Fonzo, "Traffic and mobility analysis of hierarchical cellular communication networks," IEEE Trans. Veh. Technol., vol. 52, no. 4, pp. 931-946, July 2003.

[12] A. Papoulis, Probability, Random Variables and Stochastic Processes, 3rd ed. Mc Graw Hill, 1991.

[13] C. Jedrzycki and V. Leung, "Probability distribution of channel holding time in cellular telephony systems," in Proc. IEEE Veh. Technol. Conf., GA, May 1996, pp. 247-251.

[14] E. Kreyszig, Advanced Engineering Mathematics, 6th ed. Wiley \& Sons, 1987.

[15] L. Kleinrock, Queuing Systems, Vol. 2nd: Computer Applications. Wiley \& Sons, 1985.

[16] F. Khan and D. Zeghlache, "Effect of cell residence time distribution on the performance of cellular mobile networks," in Proc. IEEE VTC'97, Phoenix, May 1997, pp. 949-953. 